\title{
TRANSONIC WING SHAPE OPTIMIZATION USING A GENETIC ALGORITHM
}

\author{
Terry L. Holst and Thomas H. Pulliam \\ NASA Ames Research Center \\ Numerical Aerospace Simulations Division, Applications Branch \\ Moffett Field, CA 94035
}

\begin{abstract}
A method for aerodynamic shape optimization based on a genetic algorithm approach is demonstrated. The algorithm is coupled with a transonic full potential flow solver and is used to optimize the flow about transonic wings including mult-objective solutions that lead to the generation of pareto fronts. The results indicate that the genetic algorithm is easy to implement, flexible in application and extremely reliable.
\end{abstract}

\section{Background}

Numerical methods for optimizing aerodynamic performance have been studied for many years. Perhaps the most widely used general approach involves the computation of sensitivity gradients. These methods-called gradient methods-have been utilized to produce optimal aerodynamic performance in a wide variety of different forms. The reliability and success of gradient methods generally requires a smooth design space and the existence of only a single global extremum.

In contrast to gradient methods, genetic algorithms (GA) offer an alternative approach with several attractive features. The basic idea associated with the GA is to search for optimal solutions using an analogy to the theory of evolution. During solution iteration (or "evolution" using GA terminology) the decision variables or "genes" are manipulated using various operators (selection, crossover or mutation) to create new design populations, i.e., new sets of genes. Each design is evaluated using an objectivelike "biological fitness function" to determine survivability. Constraints can easily be included in this approach either by direct inclusion into the fitness function or by special gene constraints. For example, if a design violates a constraint, its fitness is set to zero, i.e., it does not survive to the next evolution level.

Because GA optimizátion requires no gradients, it does not need sensitivity derivatives. It theoretically works well in non-smooth design spaces containing several or perhaps many local extrema. It is also an attractive method for multi-objective design applications offering the ability to compute socalled "pareto optimal sets" instead of the limited single design point traditionally provided by other methods. A disadvantage of the GA approach is expense. In general, the computational effort required for a GA algorithm exceeds that required by a gradient-based optimization method by an order of magnitude or more.

\section{Approach}

A GA-based optimization method is used to perform aerodynamic shape optimizations for wings at transonic cruise. Both single- and multi-objective optimizations are considered. The geometric parameterization used in the present study comes from Sobieczky. ${ }^{1}$ In this approach, each defining airfoil section is characterized by ten parameters plus a local twist angle. A graphical description of these parameters is presented in Fig.1. Typical maximum and minimum user-specified constraints for each of these parameters are given in Table 1. A wing is constructed by connecting each of the airfoil defining stations together using linear lofting. Using GA terminology, each geometric parameter is called a gene, and each set of parameters that fully specifies a unique wing geometry is called a chromosome. A set of chromosomes is called a generation or a population. The present $\mathrm{GA}^{2}$ begins by utilizing a random number generator to establish an initial population of chromosomes with random gene values that lie between the maximum and minimum constraints. Each chromosome is then evaluated using the TOPS full potential solver ${ }^{3}$ to determine fitness-e.g., the value of pressure drag for a fixed level of lift.

A new generation is established by first ranking the old chromosomes. For example, if $L / D$ maximization is sought, the design or chromosome with the largest value of $L / D$ is ranked number one, the 

chromosome with the second largest value of L/D is ranked number two and so on. Next, a selection operator is utilized to determine which chromosomes are carried forward to the next generation. Those chromosomes with the highest rank are typically selected multiple times, those with moderate rank a small number of times and those with lowest rank are not selected at all, i.e., they "die off." The selection process continues until NC chromosomes have been selected. NC is the user-specified number of chromosomes that remains fixed from one population to the next.

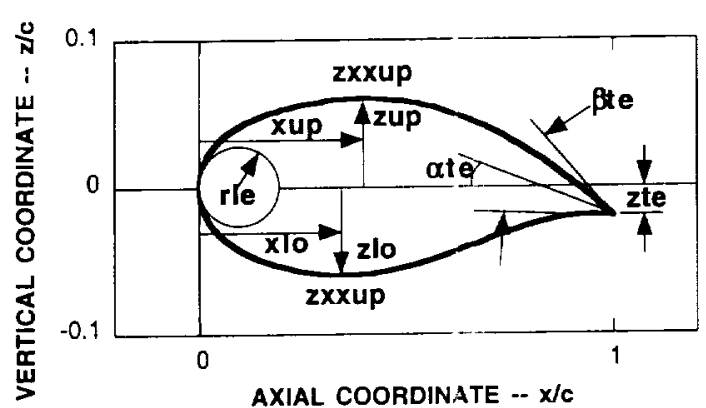

Fig. 1 Airfoil parameterization used for gene encoding.

\begin{tabular}{|l|l|l|}
\hline Parameter & Minimum & Maximum \\
\hline rle & 0.008 & 0.02 \\
\hline xup & 0.35 & 0.45 \\
\hline zup & 0.06 & 0.07 \\
\hline zxxup & -0.8 & -0.2 \\
\hline xlo & 0.35 & 0.45 \\
\hline zlo & -0.07 & -0.06 \\
\hline zxxlo & 0.2 & 0.8 \\
\hline zte & -0.01 & 0.01 \\
\hline ate & 0.0 & 8.0 \\
\hline Bte & 6.0 & 15.0 \\
\hline
\end{tabular}

Table 1. Maximum and minimum constraint values used for each gene.

After a new pool of NC chromosomes has been selected they are modified using one of four different modification operators: passthrough, crossover, perturbation-mutation and mutation. The passthrough operator takes the best chromosomes and passes them through to the next generation without modification. This ensures that the maximum fitness will never decrease. The crossover operator computes modified chromoscmes by averaging randomly chosen gene values from randomly chosen chromosomes. Of course, gene position is always maintained in the crossover operator. If the fifth position gene is chosen for averaging, two randomly chosen fifth position genes are averaged. The perturbationmutation and mutation operators create modified chromosomes by applying mutations to randomly chosen gene values from randomly chosen chromosomes. Again, this is done with gene position preservation. The magnitude of the modification computed by the perturbation-mutation operator is controlled by user input and is typically an order of magnitude smaller than the modifications produced by the mutation operator. The number of chromosomes modified by each operator is user-controlled by the $P$ vector. For example, $P=(0.1,0.2,0.3,0.4)$, causes $10 \%$ of all newly selected chromosomes to be passed through, $20 \%$ to be modified using crossover, $30 \%$ to be modified using perturbation-mutation and $40 \%$ to be modified using mutation.

Because the most-fit individuals-e.g., the wing designs with the best L/D values, if that is what is being optimized-are emphasized in the selection process, the overall level of solution fitness improves from generation to generation and converges to the optimal answer for a sufficiently large number of generations. A complete description of the precise GA used herein can be found in Ref. 2.

\section{Results}

Results from a single-point pressure drag minimization for an isolated wing at $M_{0}=0.84$ and $G_{L}=$ 0.45 are presented in Fig. 2. In this computation $N C=20$ and $P=0.1,0.3,0.3,0.3$. The wing planform was held fixed at $A R=6.4, T A=0.5$ and $\Lambda_{L E}=37^{\circ}$. The design space parameterization consisted of 22 genes - the ten parameters defined by Fig. 1 plus wing twist times two for the two wing defining stations-root and tip. Pressure coefficient distributions at four span stations for both the baseline and optimized wing are presented in Fig. 2. For this and all subsequent computations the baseline solution is simply obtained by averaging all genes in the design space. Thus, the baseline computation represents the solution obtained at the design space center. Note that the optimization has produced a solution with significantly reduced shock strength at all span stations relative to the baseline solution.

Also displayed in Fig. 2 are the baseline and optimized airfoil shapes for the wing root and tip-both greatly exaggerated in the $z$ direction. Note that the optimized wing root has been thinned and flattened on the upper surface and that both optimized root and tip sections have added aft camber. 


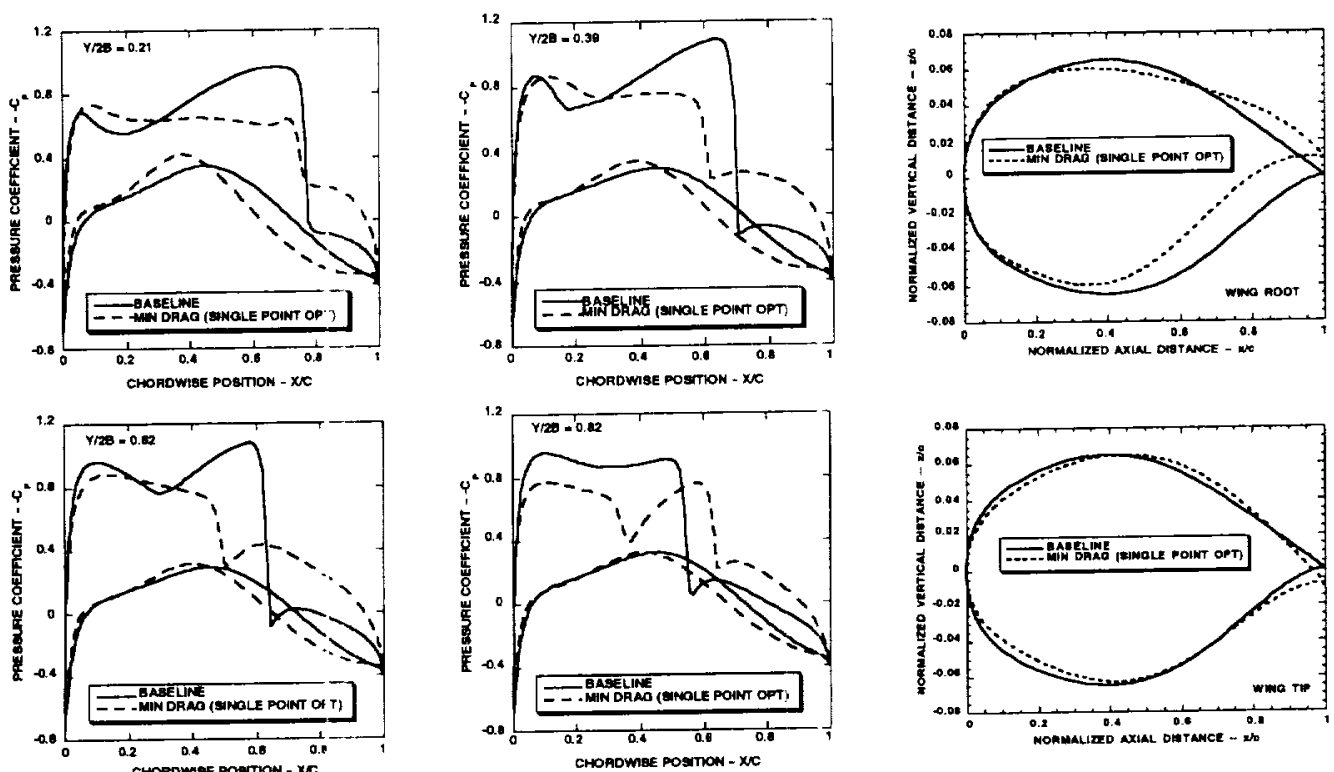

Fig. 2 Pressure coefficient distributions and airfoil sections at selected semi-span stations showing the baseline and optimized solutions for an isolated-wing drag minimization, $M_{\infty}=0.84, G=0.45, \Lambda_{L E}=37^{\circ}$, $\mathrm{AR}=6.4, \mathrm{TR}=0.5, \mathrm{NC}=20, \mathrm{~F}^{2}=(0.1,0.3,0.3,0.3), \mathrm{NG}=22$.

Figures 3 and 4 focus on GA convergence performance for the single-objective problem just discussed. Figure 3 shows the effect of population size (NC) on GA convergence. For this 22-gene aerodynamic shape optimization problem, it is clear that the use of a smaller number of chromosomes (NC $\sim 10-20$ ) produces superior GA convergence. Note that good convergence is obtained in approximately 1000 function evaluations for this problem when using an optimal value of NC.

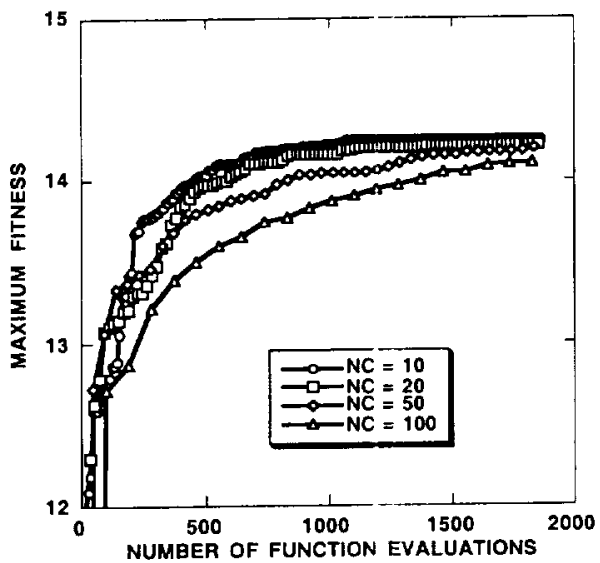

Fig. 3 GA convergence prerformance as a function of NC (GA population size) for the single-point drag minimization computation shown in Fig. 2.

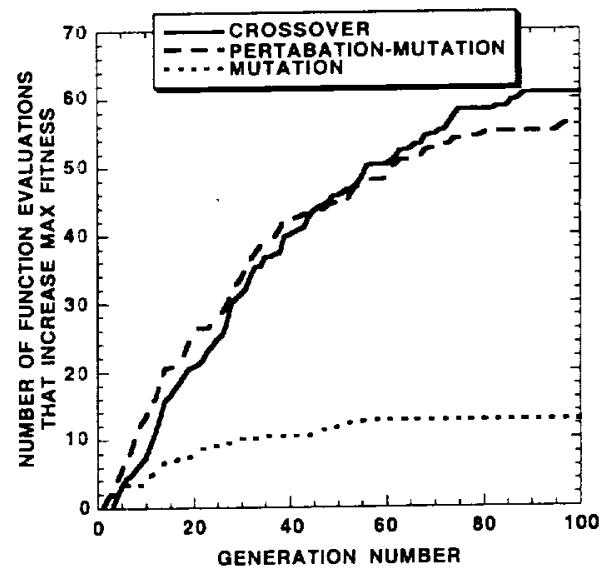

Fig. 4 Number of function evaluations that produce an increase in L/D versus generation number for the single-point drag minimization computation shown in Fig. 2.

Figure 4 shows the number of function evaluations divided among the various modification operators that produced an increase in $L D$ as a function of generation number. There are only three curves in Fig. 4 because the passthrough operator never increases the value of the objective. These 

results utilized a $P$ vector of $(0.1,0.3,0.3,0.3)$, i.e., there was an equal emphasis placed on the last three modification operators. Despite equal utilization of the last three modification operators, crossover and perturbation-mutation are more successful in producing an increase in $L / D$ than mutation. This is because the present design space is reasonably smooth and because crossover and perturbation-mutation are best suited for climbing to the top of a smooth peak-assuming that peak has already been found by at least one chromosome. Mutation, on the other hand, is primarily suited for jumping from one peak to another or in contending with design space noise, operations that are not very important for the present problem.

The next result is asscrciated with a two-point optimization problem. Genetic Algorithms are ideally suited for this type of problem and lend themselves to the direct computation of so-called "pareto optimal fronts." A pareto front is the collection of solutions in a design space, which contains all combinations of optimal solutions distributed among the two or more objectives. Pareto fronts are useful in performing engineering tradeoffs, as the complete set of optimal solutions can be viewed simultaneously. Multiobjective problems can be single disciple or multi-discipline. In the former case all objective values are determined using a single discipline analysis model. In the latter case, more than one discipline analysis model is used. More can be found on genetic algorithms including pareto fronts in Deb. ${ }^{4}$

Figure 5 shows results for a single-discipline optimization problem with two different aerodynamic objectives. The first objective is lift-to-drag ratio and the second is lift-to-drag ratio with a pitching moment penalty constraint. Specifically, these objectives are defined by

$$
O b j_{1}=\frac{1}{\left(C_{D}+C_{D, f}\right) / C_{L}}, \quad O b j_{2}=\frac{1}{\left(C_{D}+C_{D, f}\right) / C_{L}+1 / C_{M}^{2}}
$$

where $C_{D, f}$ is a constant used to approximate viscous skin fruction drag, and $C_{M}$ is the pitching moment coefficient computed about the wing root half chord position. For all cases $C_{D, f}=0.02$. The free-stream conditions, lift coefficient, wing planform and parameterization are all identical to the problem presented in Fig. 2. For this case 50 chromosomes are used in each population. A larger value of NC is used for this case because of the multi-point nature of the optimization.

The development of the pareto front for this two-point optimization computation is displayed in the lower-left portion of Fig. 5. Note the progressive improvement in the front as it moves upward and to the right with increasing generation number (IPOP value). The slowing rate of change in the front indicates the approach of convergence. Additional populations would probably produce additional small improvements in the front but at a disproportionately large computational cost. Note the significant improvement obtained over the baseline solution.

Development of a pareto front like that of Fig. 5 is of interest because it shows the effect of two or more objectives on each other, i.e., it establishes a series of optimal tradeoffs between the two objectives. In the present case the effect of a moment penalty constraint is obtained on a traditional L/D maximization. A similar wing optimization problem using a GA approach is presented in Vicini and Quagliarella. ${ }^{5}$

Also displayed in Fig. 5 are five pressure distributions plotted at a semi-span station of $39 \%$ for a number of different solutions along the pareto front. It can be seen from this set of pressure distributions that the effect of the moment contraint is to reduce the aft loading in favor of forward loading and thus to increase the shock strength. Movement of the center of pressure forward reduces the moment coefficient, and thus could result in reduced trim drag, but it causes an increase in wave drag.

The last result is associated with a multi-point optimization problem involving two different disciplines-aerodynamics and structures. The first objective is lift-to-drag ratio and the second is the inverse of the nondimensionalized structural mass. The structures model used in this computation is a simple equivalent box beam model similar to the model used in Oyama. ${ }^{6}$ Given a set of loads from the aerodynamics routine, the structures model computes the minimum mass box beam that will support that load with a factor of safety of two. The free-stream conditions, lift coefficient, wing planform and parameterization are identical to that of the last problem. For this case 32 chromosomes are used, so as to take advantage of a 32 processor parallel computer. This speeds up turn around time as the present approach is easily parallelized 


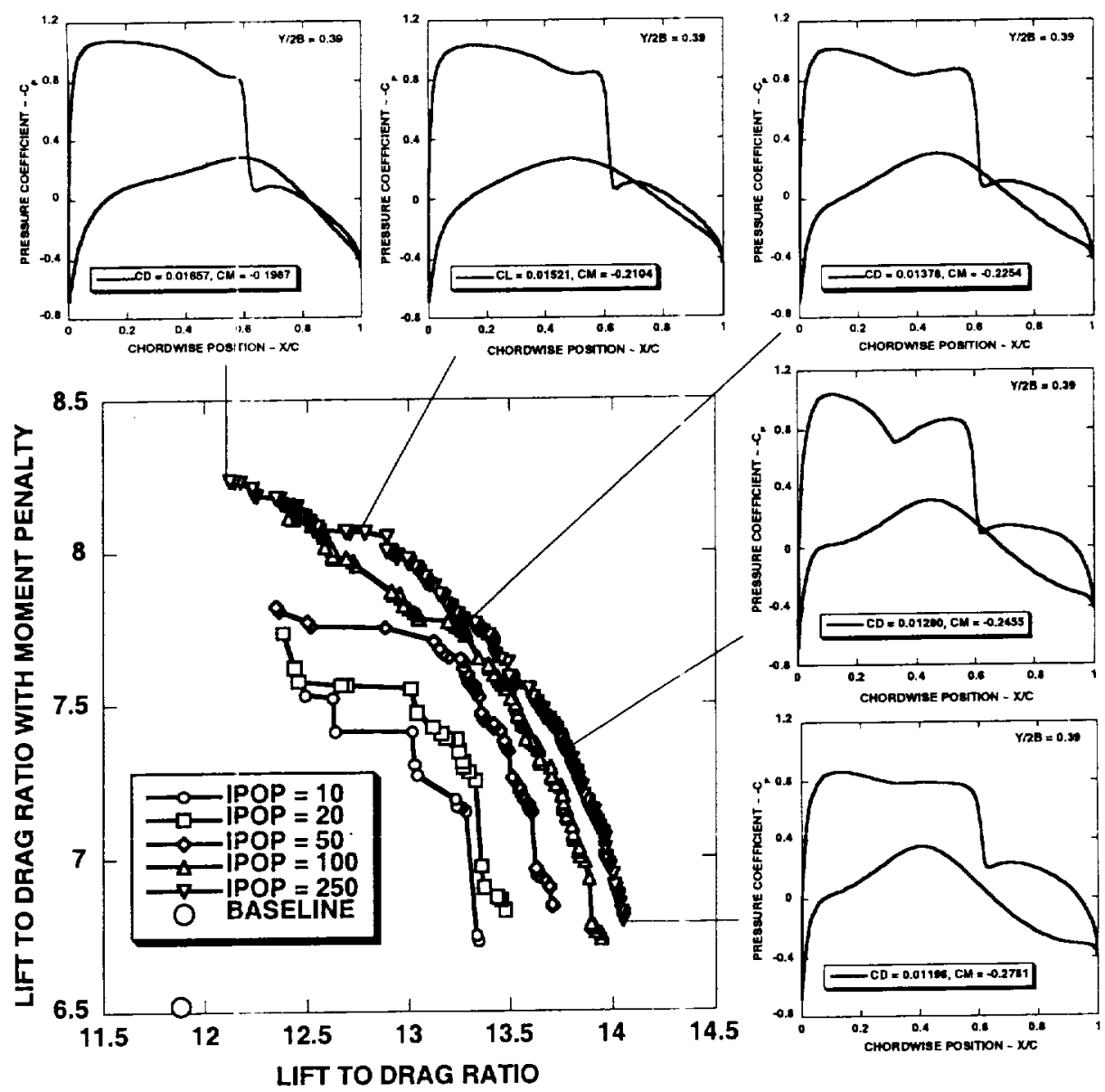

Fig. 5 Summary of results for a two-point aerodynamic optimization that shows pareto front convergence for a transonic wing optimization and selected pressure distributions along the pareto front, $M_{\infty}=0.84, C_{L}$ $=0.45, \Lambda_{\mathrm{LE}}=37^{\circ}, \mathrm{AR}=6.4, \mathrm{TR}=0.5, \mathrm{NC}=50$ and $\mathrm{NG}=22$.

The development of the pareto front for this two-point optimization computation is displayed in the lower-left portion of Fig. 6 . Note the progressive improvement in the front as it moves upward and to the right with increasing generation number. The slowing rate of change in the front indicates the approach of convergence. The last two plotted fronts are nearly on top on each other.

Also displayed in Fig. 6 are pressure distributions plotted at semi-span stations of 27 and $75 \%$ for three different solutions along the pareto front. It can be seen from this set of results that the maximum LD point on the pareto front produces a nearly shock-free pressure distribution while the minimum structural mass point has a strong shock. This is because the latter pareto point is achieved by thickening the wing root section-thus reducing the structural mass required to sustain the wing root bending moment. The minimum structural mass point is also associated with an inboard shift in wing loading. This reduces the wing-root bending-moment moment arm and further reduces the structural mass required to support the aerodynamic load.

\section{Conclusions}

A genetic algorithm (GA) has been used to perform single- and multi-objective shape optimizations for isolated wing geometries in the transonic speed regime. The computed results included a single-point drag minimization, a two-point aerodynamic optimization and a two-point multidiscipline 

optimization involving aerodynamic drag and structural mass minimization. The GA performed surprisingly well on all cases presented, demonstrating wide applicability.

For multi-point problerns an optimal set of solutions called the pareto front was generated. Pareto fronts are useful in performing engineering tradeoffs, as the complete set of optimal solutions can be viewed simultaneously.

GA optimization is quite easy to setup providing problem parameterization and function evaluation mechanisms are available. It is theoretically applicable for most engineering problems even when the design space is non-smooth.

Despite good performance from the GA, it is recognized that other methods, when they are applicable, are more efficient. The strength of genetic algorithms lies in their ability to provide optimal solutions for non-smooth or multi-modal design spaces and for multi-objective problems. This will be the focus of future work.

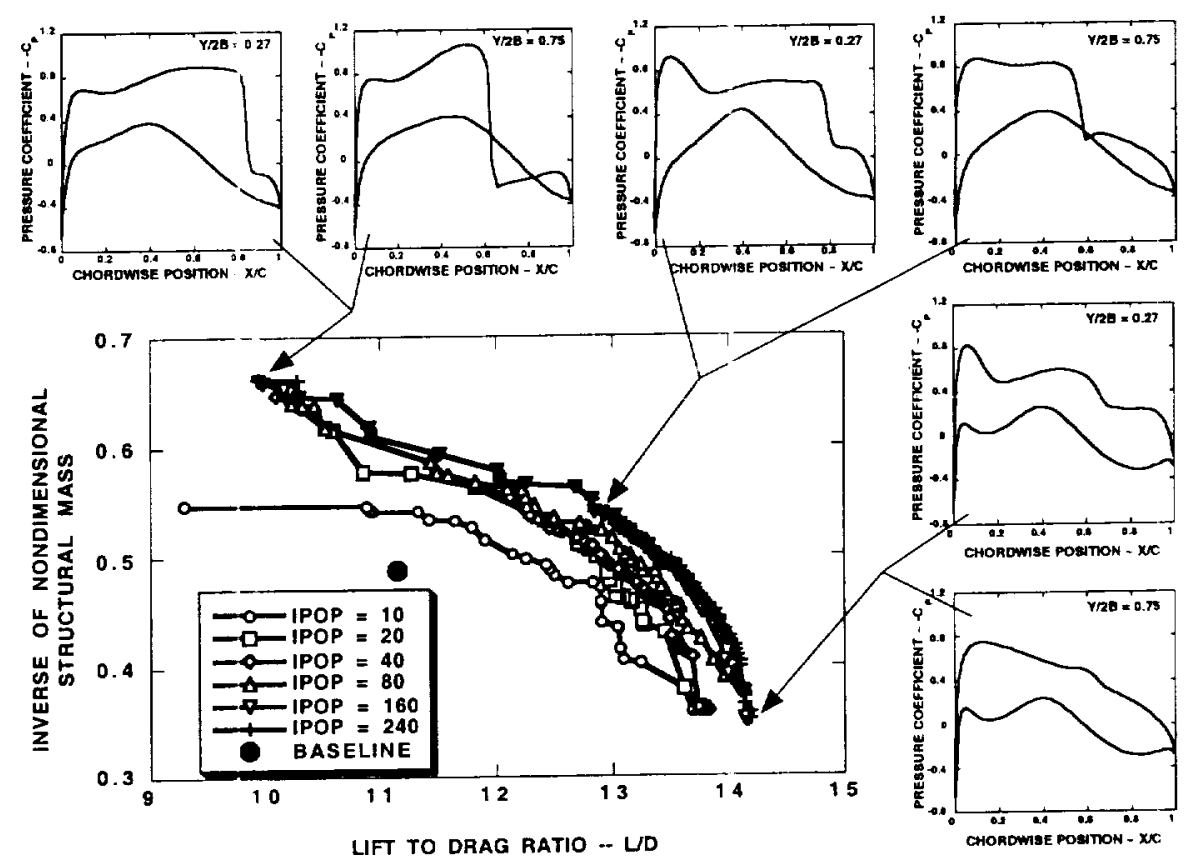

Fig. 6 Summary of results for a two-point aerodynamic/structures transonic wing optimization that shows pareto front convergence and selected pressure distributions along the pareto front, $M_{\infty}=0.84, C_{L}=0.45$, $\Lambda_{\mathrm{LE}}=37^{\circ}, \mathrm{AR}=6.4, \mathrm{TR}=0.5, \mathrm{NC}=32$ and $\mathrm{NG}=22$.

\section{References}

1. Sobieczky, H., "Parametric Airfoils and Wings," Recent Development of Aerodynamic Design Methodologies-Inverse [lesign and Optimization, Friedr. Vieweg \& Sohn Verlagsgesellschaft mbH, Braunschweig/Wiesbaden, Germany, 1999, pp. 72-74.

2. Holst, T. L. and Pulliam, T., "Aerodynamic Shape Optimization Using a Real-Number-Encoded Genetic Algorithm," AIAA Paper No. 2001-2473, June 2001.

3. Holst, T. L., "Multizone Chimera Algorithm for Solving the Full-Potential Equation," J. of Aircraft, Vol. 35, No. 3, May-June 1993, pp. 412-421.

4. Deb, K., "Multi-Objective Genetic Algorithms: Problem Difficulties and Construction of Test Problems," Evolutionary Computation, Vol. 7, No. 3, 1999, pp. 205-230.

5. Vicini, A. and Quagliarella, D., "Airfoil and Wing Design Through Hyprid Optimization Strategies," AIAA Paper No. 98-2729, 1998.

6. Oyama, A., "Wing Desigr Using Evolutionary Algorithms," PhD Thesis, Dept. of Aeronautics and Space Engineering, Tohcku University, Senadi, Japan, March 2000. 
